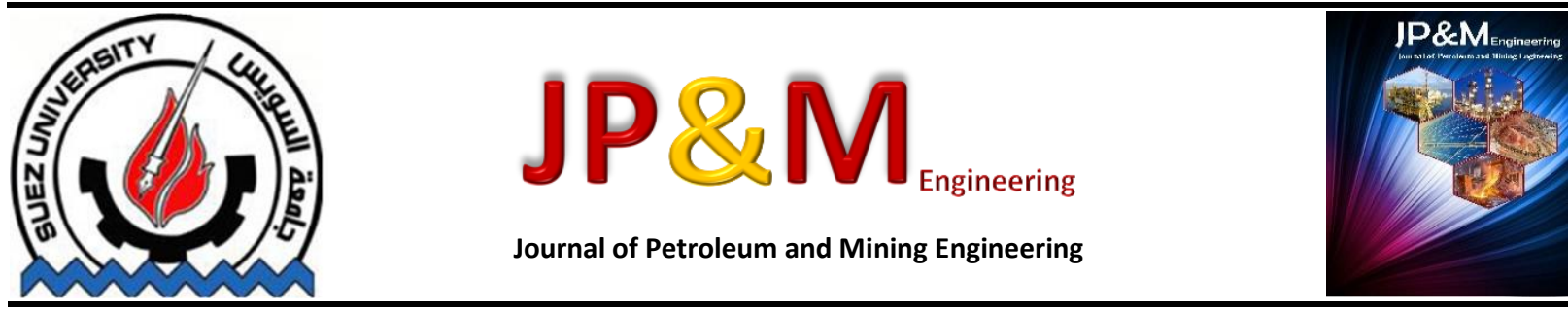

\title{
Characterization of Calcium Carbonate Rocks, East El Minya Deposits for Possibility Uses as Industrial Raw Materials
}

\author{
A.M Abdelaal ${ }^{\mathrm{a}}$, S Sameah ${ }^{\mathrm{b}}$ and A Ahmed ${ }^{\mathrm{a}}$ \\ ${ }^{a}$ Faculty of Petroleum and Mining Eng., Suez University. \\ ${ }^{\mathrm{b}}$ The Egyptian Mineral Resources Authority, Cairo, Egypt
}

\begin{abstract}
This study deals with prospecting and evaluation of the calcium carbonate rock deposits the East El-Minya, near the Nile Valley-Upper Egypt for a proper industrial use. The study covered the area located between the following points: G10 (28ㅇ 22'

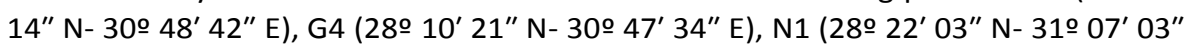

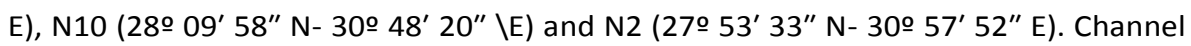
samples were collected from trenches and quarries for valuation. Each sample was prepared by crushing and grinding for different types of analyses which were carried out using XRF for chemical composition and the brightness of the materials were also measured. The obtained results show that the studied area for limestone observed in the Middle Eocene deposits and has high quality compassion; purities and whiteness. According to the obtained results, the limestone deposits are suitable for chemical industry and others industrial uses where the specification needed for these industries exist.
\end{abstract}

Keywords

Limestones; calcium carbonate rock; industry uses; Egypt; East El-Minya.

\section{Introduction}

Calcium carbonate (Limestone), as a sedimentary calcareous rock, mostly composed of calcite with some gangue minerals such as quartz, feldspar, iron oxides and mica. Limestones ore considered as a very important industrial mineral and the major industries which consume limestone are: cement production, extraction metallurgy (iron and steel making), manufacturing (glass, ceramics, blackboard chalk, food processing, paper making as a filler and a coating pigment, leather, insulation and $\mathrm{pH}$ control), agriculture (fertilizers, fungicides, animal feed), construction/architecture (mortar, cement, whitewash, building stone, concrete wares as pavingstones, tubes, sewage-tanks), plastics (plasticised and rigid PVC, unsaturated polyesters, polypropylene, polyethylene, rubber, foamed latex carpet-backings, sealants and adhesives), environment (flue gas desulphurisation, drinking water treatment, waste water treatment and forest and lake liming for the neutralisation of acid rain) purposes $[1,2]$. The constituents of Limestone ore as a raw material for different industrial uses play a significant role in the cost-effectiveness. The most significant constituents of limestones as above mentioned are calcium oxide (CaO), alumina (Al2O3), iron oxide (Fe2O3), magnesium (MgO), and silica ( $\mathrm{SiO} 2)[1,3]$. Industrial specifications of calcium carbonate (\%) for some different uses are shown in Table 1 [4-10]. Some research work has been carried out on the evaluation of Egyptian carbonate rocks for construction purposes include geotechnical analyses of limestone specimens from north to south Egypt. The first comprehensive work was performed by Tame and Edet, on the suitability of Egyptian limestone for cement manufacture [11-15]. Calcium carbonate (Limestone) rocks cover wide areas throughout Egypt map forming ridges and plateaus. In the majority of these areas the limestone rocks found on the surface or in shallow depth to include significant reserves of high grade material [16].

Limestone purity was classified according to the weight percentage of $\mathrm{CaCO}_{3}, \mathrm{CaO}, \mathrm{MgO}, \mathrm{SiO}_{2}$ and $\mathrm{Fe}_{2} \mathrm{O}_{3}$ as indicated in Table 2 below [17].

Deposits of limestone in the Nile Valley extent nearly from north to south along the Valley comprising Manfalut, El-Minya, Samulut and Mokattam Formations. While its deposits in Canal Governorates lie in three areas; Gebel Aukhedu, EIShallufa and El Sadat. Also, the limestone rocks deposits found in west Alexandria in the Abu Sir, Sidi Krier, Borg El-Arab and El-Hammam. Several economic limestone deposits are also found in North Sinai, for example; Al Geham, Rissan Aneiza, ElMestan - Umm Mofrouth, Gebel El-Raghaway, Gabal El Maghara and others [16]. The work here deals with the calcium carbonate mineral exploration for 
different industrial uses. The study area occupies eastern parts of the Nile bank at El Mania Governorate. It is located between latitudes 280 04

Table 1 Chemical properties and brightness of commercially available calcium carbonate [4-10].

\begin{tabular}{|c|c|c|c|c|c|c|c|c|}
\hline \multirow[t]{2}{*}{ Properties } & \multicolumn{2}{|r|}{ Paint } & \multicolumn{2}{|r|}{ Paper } & \multicolumn{2}{|r|}{ Plastic } & \multicolumn{2}{|c|}{ Food \& pharmaceutic } \\
\hline & Mean & Range & Mean & Range & Mean & Range & Mean & Range \\
\hline $\mathrm{CaCO}_{3}(\%)$ & 97.80 & $92-99.35$ & 98.46 & $96-99.35$ & 97.97 & $92-99.35$ & 98.29 & $97-99.5$ \\
\hline $\mathrm{CaO}(\%)$ & 54.80 & $51.55-55.67$ & 55.17 & $53.79-55.67$ & 54.89 & $51.55-55.67$ & 55.07 & $54.35-55.75$ \\
\hline MgO (\%) & 0.42 & $0.15-1.2$ & 0.45 & $0.15-1.2$ & 0.46 & $0.15-1.2$ & 0.29 & $0.24-0.42$ \\
\hline $\mathrm{SiO}_{2}(\%)$ & 0.72 & $0.05-4.5$ & 0.11 & $0.05-0.4$ & 0.46 & $0.05-4.5$ & 0.11 & $0.1-0.12$ \\
\hline $\mathrm{Fe}_{2} \mathrm{O}_{3}(\%)$ & 0.04 & $0.01-0.1$ & 0.04 & $0.01-0.1$ & 0.04 & $0.01-0.1$ & 0.06 & $0.011-0.1$ \\
\hline Brightness (\%) & \multirow{2}{*}{\multicolumn{2}{|c|}{$\begin{array}{l}78-99 \\
100\end{array}$}} & 96.7 & $93.5-99$ & 92.7 & $75-98.1$ & 96.5 & $90-99$ \\
\hline No. of datasheets & & & \multicolumn{2}{|r|}{35} & \multicolumn{2}{|c|}{88} & \multicolumn{2}{|c|}{34} \\
\hline \multirow[t]{2}{*}{ Properties } & \multicolumn{2}{|c|}{ Ceramic } & \multicolumn{2}{|r|}{ Rubber } & \multicolumn{2}{|c|}{ Adhesives \& sealants } & \multicolumn{2}{|c|}{ Agriculture \& animal feed } \\
\hline & Mean & Range & Mean & Range & Mean & Range & Range & Mean \\
\hline $\mathrm{CaCO}_{3}(\%)$ & 99.05 & $98.8-99.35$ & 97.37 & $92-99.35$ & 97.48 & $92-99.35$ & 95.55 & $92-99.35$ \\
\hline $\mathrm{CaO}(\%)$ & 55.50 & $55.36-55.67$ & 54.56 & $51.55-55.67$ & 54.62 & $51.55-55.67$ & 54.09 & $51.55-55.67$ \\
\hline MgO (\%) & 0.27 & $0.22-0.38$ & 0.62 & $0.15-1.2$ & 0.54 & $0.15-1.2$ & 0.66 & $0.22-0.96$ \\
\hline $\mathrm{SiO}_{2}(\%)$ & 0.08 & $0.06-0.12$ & 0.82 & $0.05-4.5$ & 1.14 & $0.05-4.5$ & 1.28 & $0.06-4.5$ \\
\hline $\mathrm{Fe}_{2} \mathrm{O}_{3}(\%)$ & 0.03 & $0.02-0.044$ & 0.04 & $0.01-0.1$ & 0.02 & $0.01-0.1$ & 0.01 & $0.037-0.1$ \\
\hline Brightness (\%) & 95.6 & $95.3-96$ & 90.6 & $75-98.5$ & 91.0 & $75-98$ & 81.8 & $70-95.5$ \\
\hline No. of datasheets & \multicolumn{2}{|r|}{14} & \multicolumn{2}{|r|}{51} & \multicolumn{2}{|r|}{65} & \multicolumn{2}{|r|}{14} \\
\hline
\end{tabular}

Table 2 Classification of the Limestones ore by purity.

\begin{tabular}{|c|l|c|c|c|c|}
\hline Purity classification & CaCO3 \% wt. & CaO \% wt. & MgO \% wt. & SiO2 \% wt. & Fe2O3 \% wt. \\
\hline Very high purity & $>98.5$ & $>55.2$ & $<0.8$ & $<0.2$ & $<0.05$ \\
\hline High purity & $97-98.5$ & $54.3-55.2$ & $0.8-1.0$ & $0.2-0.6$ & $0.05-0.1$ \\
\hline Medium purity & $93-97$ & $52.4-54.3$ & $1.0-3.0$ & $0.6-1.0$ & $0.1-1.0$ \\
\hline Low purity & $85-93$ & $47.6-52.4$ & $>3.0$ & $<2.0$ & $>1.0$ \\
\hline Impure & $<85.0$ & $<47.6$ & & $>2.0$ & \\
\hline
\end{tabular}

\section{Geography, Geology and Geomorphology of The Study}

\section{Geography}

The study area is accessible through a net of asphaltic roads and desert tracks, which almost cross the area parallel to the Nile Valley. The main highway in the area is Aswan Cairo asphaltic road as well as new military desert asphaltic roads extends from Cairo to El Mania crossing the eastern desert. Several asphaltic roads and desert tracks connect with these roads i.e. Al Shiekh Fadel - Ras Garb asphaltic road. It connects between the main towns of Upper Egypt from Cairo to Aswan and Red Sea. A number of secondary paved roads are also branched from the main high way. Also the area covered by numerous unpaved roads and desert tracks [18-23]. Geology The exposed limestone rocks in this area belong to the Lower, the Middle Eocene, and the Quaternary sediments [18]. The Eocene sediments, which, form the most of the studied area around the Nile banks of El Mania was described and studied by many authors [18-23].

\section{Geomorphology}

Geomorphic units include; High Mountains:- The high mountains are represented by Gabal El-Abid, Gabal Qarara, Gabal Daba, Gabal El -Ahmar, Gabal El Teir. The maximum elevations, $278 \mathrm{~m}$ at Gabal Daba, which is western end of El-Mirier, scarp. All these mountains consisted of limestone, marl and clay rocks of Eocene age; Wadies:- The wadis intersecting $00^{\prime \prime}$ and 280 25' north and from Nile Valley to longitudes $30 \circ 35^{\prime}$. the studied area east El-Minya from north to south are; Wadi El Mhashm, Wadi El Harafish,Wadi Sariria, Wadi Garf El Deir, Wadi El-Tahnawy and Wadi Omarn. All these wadies flow toward the Nile; Fanglomerate: - The fanglomerate is of limited extension present in some localities overlooking the Nile valley crossing the Eocene and laid down on the flood plain area. It consists of boulder and gravels of limestone, chart and quartz of rounded to sub rounded with matrix of reddish brown coarse sands; Flood plain and cultivated land: - The flood plain varies in width from place to another. The flood plain is made up of sand and gravel. The cultivated land included the Nile mud and silt of the flood plain adjacent to the River Nile. It varies in width from few kilometers at east Beni Mazar and Maghagha area. The level of the cultivated land varies in elevation from $25 \mathrm{~m}$ to $80 \mathrm{~m}$ [19-23].

\section{Methods and Exploration Procedure.}

This study is based on the collected samples from four measured stratigraphic column sections within the area under investigation as the following: A 29 channel samples were taken from excavating trenches and quarries along the limestone plateau of the Samalut Formation East of El-Minya in the South toward East of Samalut in the North (Figure 1). 


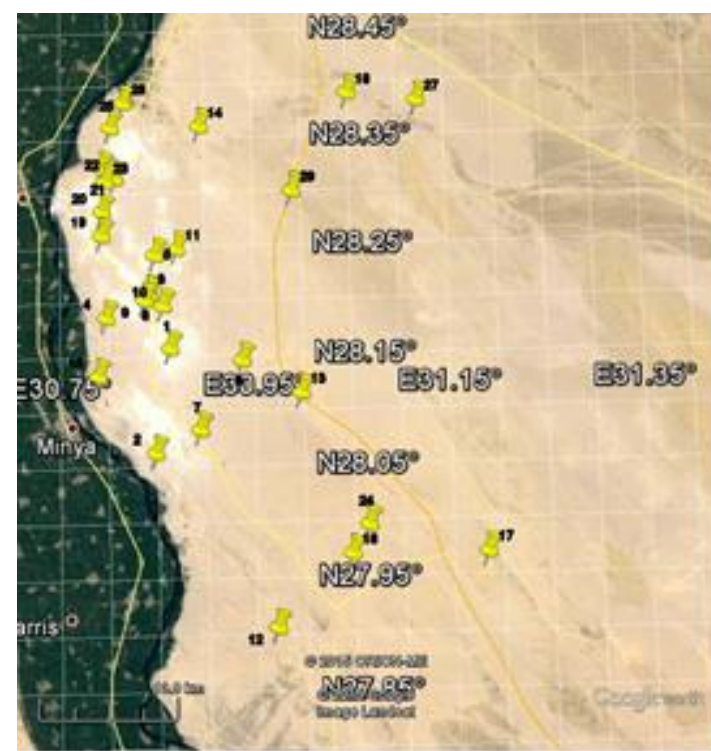

Figure $1 \mathrm{~A}$ plan of the area under investigation.

\section{Results and Discussions}

The chemical analysis of calcium carbonate rocks from XRF results are indicated in Tables 3 and 4 .

As seen from these Tables ( 3 and 4 ) above the majority of the study area occupies eastern parts of the Nile bank at El-Minya Governorate, that embraced between latitudes $28004^{\prime} 00^{\prime \prime}$ and 28 25' north and from Nile Valley to longitudes 30 o $35^{\prime}$ the limestone deposit ranked as a high to very high purity type. For more detailed explanation, the assay contour map technique was used to present the results for each assay item of limestone constituents. By using GIS-Arc view software the limestone assay results were presented in Fig. 2 (1- $\mathrm{CaO} ; 2-\mathrm{CaCO}_{3} ; 3-$ $\mathrm{K}_{2} \mathrm{O}$; 4- $\mathrm{Na}_{2} \mathrm{O}$; 5- $\mathrm{P}_{2} \mathrm{O}_{5}$; 6- Cl; 7- $\mathrm{SO}_{3}$; 8- LOI; 9- $\mathrm{Fe}_{2} \mathrm{O}_{3}$; 10- $\mathrm{Al}_{2} \mathrm{O}_{3} ; 11-\mathrm{MgO} ; 12-\mathrm{SiO}_{2}$ ). As indicated in Figure 2 (1 to 12 ) below. Figure 2-1 shows the percentage of average assay of $\mathrm{CaO}$ content in limestone deposits. Whenever, as we go towards the NE of the area, the $\mathrm{CaO} \%$ increases to reach $55.8 \%$. We find $\mathrm{CaO} \%$ content in the majority of limestone deposits in the area under investigation about $55.3 \%$. Figure 2-2 shows the percentage of $\mathrm{CaCO}_{3}$ content in limestone deposits. The $\mathrm{CaCO}_{3} \%$ content in limestone deposits as indicated in the contour map; Figure 2-2 shows that, an average assay of $\mathrm{CaCO}_{3} \%$ was about $98.6 \%$ and increases in the direction towards the NE of the area up to $99.5 \%$. As indicated in Figure $2-3, \mathrm{~K}_{2} \mathrm{O} \%$ in limestone deposits covered the most of the area was about $0.03 \%$. Also this Figure shows that, as the $\mathrm{CaCO}_{3} \%$ increased the average assay of $\mathrm{K}_{2} \mathrm{O} \%$, however, ranges from $0.01 \%$ to $0.1 \%$ by weight.

Figure 2-4, shoes the percentage of the average assay of $\mathrm{Na}_{2} \mathrm{O}$ content in limestone deposits. As seen from the Figure, that, the percentage of $\mathrm{Na}_{2} \mathrm{O}$ varied from $0.05 \%$ at both of NW and SE of the investigated area to $0.1 \%$ at NE and SW.
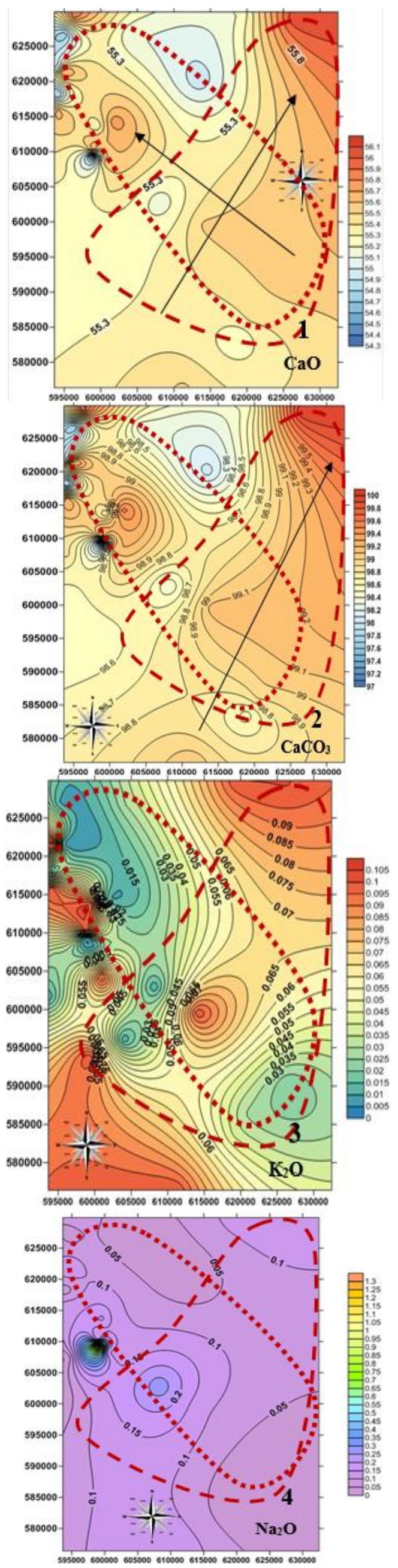
Table $\mathbf{3}$ Chemical analysis results of calcium carbonate samples obtained by XRF.

\begin{tabular}{|c|c|c|c|c|c|c|c|c|c|c|c|c|c|c|c|c|}
\hline \multicolumn{2}{|c|}{ Location } & \multirow{2}{*}{ 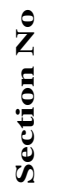 } & \multirow{2}{*}{ 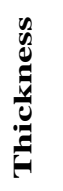 } & \multirow{2}{*}{$\begin{array}{l}\delta^{0} \\
\hat{n} \\
\dot{n}\end{array}$} & \multirow{2}{*}{$\begin{array}{l}\delta^{\circ} \\
0 \\
2 \\
2\end{array}$} & \multirow{2}{*}{ 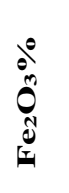 } & \multirow{2}{*}{$\begin{array}{l}0 \\
0 \\
\Sigma\end{array}$} & \multirow{2}{*}{$\begin{array}{l}8 \\
0 \\
\sum 0 \\
\sum\end{array}$} & \multirow{2}{*}{$\begin{array}{l}0 \\
0 \\
0\end{array}$} & \multirow{2}{*}{$\begin{array}{l}0 \\
0 \\
\tilde{Z} \\
\bar{Z}\end{array}$} & \multirow{2}{*}{$\begin{array}{l}0 \\
0 \\
\underline{N}\end{array}$} & \multirow{2}{*}{$\begin{array}{l}d \\
0^{\circ} \\
0^{n}\end{array}$} & \multirow{2}{*}{$\frac{\theta^{0}}{u}$} & \multirow{2}{*}{$\stackrel{\theta}{0}$} & \multirow{2}{*}{$\begin{array}{l}\dot{0} \\
\dot{\dot{j}} \\
\dot{j}\end{array}$} & \multirow{2}{*}{$\overbrace{0}^{\infty}$} \\
\hline Lat. & Long. & & & & & & & & & & & & & & & \\
\hline \multirow{2}{*}{$28^{\circ} 08^{\prime} 37^{\prime \prime}$} & $30^{0} 51^{\prime} 31^{\prime \prime}$ & 1 & 8.0 & 01 & 0.1 & 0.05 & 0.01 & & 55.47 & & 0.1 & 0.01 & 0.04 & 0.58 & 43.03 & 99.00 \\
\hline & 3051 & & 7.0 & 0.45 & 0.1 & 0.14 & 0.01 & 0.3 & 55.02 & 0.12 & 0.1 & 0.00 & 0.01 & 0.1 & 43.56 & 98.20 \\
\hline & & ? & 8.0 & 0.19 & 0.1 & 0.11 & 0.01 & 0.32 & 55.21 & 0.12 & 0.1 & 0.01 & 0.1 & 0.07 & 43.61 & 98.54 \\
\hline & & 2 & 4.0 & 0.06 & 0.1 & 0.05 & 0.01 & 0.27 & 55.23 & 0.14 & 0.1 & 0.01 & 0.1 & 0.15 & 43.71 & 98.57 \\
\hline $28^{\circ} 11^{\prime} 41^{\prime \prime}$ & $30^{\circ} 50^{\prime} 15^{\prime \prime}$ & 3 & 9.0 & 0.1 & 0.1 & 0.03 & 0.01 & 0.29 & 55.67 & 0.14 & 0.1 & 0.01 & 0.01 & 0.1 & 43.64 & 99.36 \\
\hline & & 4 & 8.0 & 1.0 & 0.09 & 0.08 & 0.01 & 0.01 & 55.60 & 0.01 & 0.03 & 0.07 & 0.01 & 001 & 43.15 & 99.25 \\
\hline & & 4 & 25.0 & 1.0 & 0.06 & 0.02 & 0.01 & 0.01 & 55.49 & 0.01 & 0.02 & 0.01 & 0.01 & 0.01 & 43.52 & 99.04 \\
\hline & & 5 & 8.0 & 0.03 & 0.1 & 0.07 & 0.01 & 0.33 & 55.19 & 0.27 & 0.1 & 0.02 & 0.08 & 0.24 & 43.33 & 98.50 \\
\hline & & 5 & 7.0 & 0.1 & 0.1 & 0.02 & 0.01 & 0.30 & 55.65 & 0.20 & 0.1 & 0.01 & 0.04 & 0.03 & 43.56 & 99.32 \\
\hline $28^{\circ} 07^{\prime} 54^{\prime \prime}$ & ${ }^{0} 55^{\prime} 57^{\prime \prime}$ & 6 & 4.0 & 0.03 & 0.01 & 0.01 & 0.01 & 0.07 & 55.12 & 0.29 & 0.01 & 0.01 & 0.33 & 0.25 & 43.59 & 98.39 \\
\hline $28^{\circ} 04^{\prime} 11^{\prime \prime}$ & $30^{\circ} 53^{\prime} 19^{\prime \prime}$ & 7 & 3.0 & 0.02 & 0.01 & 0.01 & 0.01 & 0.07 & 55.26 & 0.15 & 0.01 & 0.01 & 0.17 & 0.63 & 43.42 & 98.64 \\
\hline $28^{\circ} 11^{\prime} 34^{\prime \prime}$ & $30^{\circ} 50^{\prime} 15^{\prime \prime}$ & 8 & 4.0 & 0.03 & 0.01 & 0.01 & 0.01 & 0.07 & 53.97 & 1.71 & 0.01 & 0.01 & 1.94 & 0.14 & 41.90 & 96.34 \\
\hline $28^{\circ} 11^{\prime} 16^{\prime \prime}$ & $30^{\circ} 49^{\prime} 08^{\prime \prime}$ & 9 & 4.0 & 0.02 & 0.01 & 0.01 & 0.01 & 0.06 & 54.80 & 0.88 & 0.01 & 0.01 & 0.99 & 0.12 & 42.80 & 97.82 \\
\hline $28^{\circ} 10^{\prime} 57^{\prime \prime}$ & $30^{\circ} 51^{\prime} 05^{\prime \prime}$ & 10 & 3.0 & 0.03 & 0.01 & 0.01 & 0.01 & 0.06 & 55.53 & 0.18 & 0.01 & 0.01 & 0.20 & 0.18 & 43.67 & 99.12 \\
\hline $28^{\circ} 14^{\prime} 03^{\prime \prime}$ & $30^{\circ} 51^{\prime} 59^{\prime \prime}$ & 11 & 4.0 & 0.02 & 0.01 & 0.01 & 0.01 & 0.07 & 55.78 & 0.12 & 0.01 & 0.01 & 0.14 & 0.03 & 43.58 & 99.57 \\
\hline $27^{0} 53^{\prime} 33^{\prime \prime}$ & $30^{\circ} 57^{\prime} 52^{\prime \prime}$ & 12 & 10.0 & 0.1 & 0.1 & 0.04 & 0.01 & 0.31 & 55.52 & 0.14 & 0.1 & 0.01 & 0.1 & 0.1 & 43.73 & 99.09 \\
\hline $28^{\circ} 06^{\prime} 06^{\prime \prime}$ & ${ }^{0} 59^{\prime} 33^{\prime \prime}$ & 13 & 8.0 & 0.1 & 0.1 & 0.03 & 0.01 & 0.47 & 55.52 & 0.12 & 0.1 & 0.01 & 0.1 & 0.04 & 43.66 & 99.09 \\
\hline & & 14 & 20.0 & 1.0 & 0.12 & 0.06 & 0.01 & 0.01 & 54.51 & 0.01 & 0.04 & 0.02 & 0.01 & 1.59 & 42.83 & 97.30 \\
\hline & & 14 & 21.0 & 1.0 & 0.09 & 0.03 & 0.01 & 0.01 & 55.67 & 0.01 & 0.03 & 0.06 & 0.01 & 0.01 & 43.66 & 99.36 \\
\hline & & & 15.0 & 1.0 & 0.37 & 0.17 & 0.01 & 0.01 & 54.59 & 0.01 & 0.01 & 0.05 & 0.01 & 0.01 & 42.97 & 97.43 \\
\hline & & & 18.0 & 1.0 & 0.12 & 0.06 & 0.01 & 0.01 & 55.35 & 0.01 & 0.01 & 0.02 & 0.01 & 0.01 & 43.34 & 98.79 \\
\hline & & & 18.0 & 1.0 & 0.11 & 0.05 & 0.01 & 0.01 & 55.79 & 0.01 & 0.01 & 0.00 & 0.01 & 0.01 & 43.76 & 99.57 \\
\hline & & & 20.0 & 1.0 & 0.52 & 0.27 & 0.01 & 0.01 & 55.75 & 0.01 & 0.01 & 1.00 & 0.01 & 0.01 & 42.93 & 99.50 \\
\hline $28^{\circ} 09^{\prime} 58^{\prime \prime}$ & $30^{\circ} 48^{\prime} 20^{\prime \prime}$ & 16 & 30.0 & 1.0 & 0.07 & 0.03 & 0.01 & 0.01 & 55.36 & 0.01 & 0.03 & 0.01 & 0.75 & 0.01 & 43.50 & 98.81 \\
\hline & $31^{0} 1$ & 17 & 15.0 & 1.0 & 0.25 & 0.08 & 0.01 & 0.01 & 55.29 & 0.01 & 0.05 & 0.05 & 0.01 & 0.01 & 43.36 & 98.68 \\
\hline & & & 19.0 & 1.0 & 0.04 & 0.01 & 0.01 & 0.01 & 55.62 & 0.01 & 0.02 & 0.01 & 0.01 & 0.01 & 43.70 & 99.27 \\
\hline & & & 3.0 & 1.0 & 0.44 & 0.17 & 0.01 & 0.01 & 55.13 & 0.01 & 0.06 & 0.03 & 0.01 & 0.04 & 43.16 & 98.40 \\
\hline & & 10 & 4.0 & 1.0 & 0.08 & 0.03 & 0.01 & 0.15 & $\mathbf{5 5 . 3 0}$ & 0.01 & 0.03 & 0.01 & 0.10 & 0.01 & 43.37 & 98.70 \\
\hline $28^{\circ} 14^{\prime} 52^{\prime \prime}$ & $30^{\circ} 47^{\prime} 20^{\prime \prime}$ & 19 & 6,0 & 0.01 & 0.1 & 0.05 & 0.01 & 0.32 & 55.66 & 0.13 & 0.1 & 0.01 & 0.1 & 0.1 & 43.55 & 99.34 \\
\hline $28^{\circ} 16^{\prime} 13^{\prime \prime}$ & $0^{\circ} 47^{\prime} 22^{\prime \prime}$ & 20 & 5.0 & 0.07 & 0.03 & 0.05 & 0.01 & 0.05 & 54.71 & 0.02 & 0.01 & 0.01 & 0.01 & 0.03 & 43.40 & 97.65 \\
\hline & & & 4.0 & 0.1 & 0.1 & 0.04 & 0.01 & 0.33 & 54.12 & 0.16 & 0.1 & 0.01 & 0.00 & 0.1 & 39.54 & 96.60 \\
\hline & & & 8.0 & 0.1 & 0.1 & 0.04 & 0.01 & 0.34 & 5.45 & 0.14 & 0.1 & 0.01 & 0.1 & 0.49 & 43.79 & 98.97 \\
\hline $28^{\circ} 18^{\prime} 01^{\prime \prime}$ & $30^{\circ} 48^{\prime} 11^{\prime \prime}$ & 22 & 4.0 & 0.01 & 0.01 & 0.01 & 0.01 & 0.06 & 55.69 & 0.12 & 0.01 & 0.01 & 0.14 & 0.07 & 43.71 & 99.41 \\
\hline & & & 12.0 & 0.1 & 0.1 & 0.03 & 0.01 & 0.31 & 55.75 & 0.14 & 0.1 & 0.01 & 0.1 & 0.1 & 43.64 & 99.18 \\
\hline & & & 2.0 & 0.1 & 0.1 & 0.04 & 0.01 & 0.41 & 55.25 & 0.12 & 0.1 & 0.01 & 0.1 & 0.40 & 43.56 & 98.61 \\
\hline $28^{\circ} 22^{\prime} 31^{\prime \prime}$ & $31^{\circ} 02^{\prime} 44^{\prime \prime}$ & 24 & 10.0 & 0.08 & 0.1 & 0.05 & 0.01 & 0.40 & 55.22 & 0.12 & 0.1 & 0.02 & 0.1 & 0.1 & 43.77 & 98.56 \\
\hline & & & 5.0 & 1.0 & 0.08 & 0.03 & 0.01 & 0.01 & 55.46 & 0.01 & 0.03 & 0.02 & 0.01 & 0.01 & 43.42 & 98.98 \\
\hline $28^{\circ} 20^{\prime} 51^{\prime \prime}$ & $30^{\circ} 47^{\prime} 54^{\prime \prime}$ & 25 & 4.0 & 1.0 & 0.20 & 0.06 & 0.01 & 0.01 & 55.32 & 0.01 & 0.04 & 0.04 & 0.01 & 0.01 & 43.45 & 98.73 \\
\hline & & & 3.0 & 1.0 & 0.10 & 0.02 & 0.01 & 0.01 & 55.48 & 0.01 & 0.03 & 0.04 & 0.01 & 0.01 & 43.57 & 99.02 \\
\hline & & & 5.0 & 1.0 & 0.05 & 0.02 & 0.01 & 0.01 & 55.51 & 0.01 & 0.02 & 0.02 & 0.01 & 0.01 & 43.56 & 99.07 \\
\hline $28^{\circ} 22^{\prime} 14^{\prime \prime}$ & $30^{\circ} 48^{\prime} 42^{\prime \prime}$ & 26 & 5.0 & 0.14 & 0.07 & 0.02 & 0.01 & 0.08 & 55.90 & 0.07 & 0.01 & 0.01 & 0.01 & 0.02 & 45.00 & 99.77 \\
\hline & & ? & 15.0 & 0.11 & 0.1 & 0.07 & 0.01 & 0.3 & 56.06 & 0.13 & 0.1 & 0.04 & 0.1 & 0.1 & 43.20 & 99.99 \\
\hline & & & 20.0 & 0.20 & 0.1 & 0.03 & 0.01 & 0.28 & 56.11 & 0.13 & 0.1 & 0.05 & 0.1 & 0.1 & 43.10 & 99.99 \\
\hline $28^{\circ} 05^{\prime} 53^{\prime \prime}$ & $30^{\circ} 59^{\prime} 59^{\prime \prime}$ & 28 & 2.0 & 0.1 & 0.1 & 0.04 & 0.01 & 0.30 & 55.22 & 0.12 & 0.1 & 0.01 & 0.1 & 0.61 & 43.42 & 98.56 \\
\hline $28^{\circ} 17^{\prime} 09^{\prime \prime}$ & $30^{\circ} 59^{\prime} 10^{\prime \prime}$ & 29 & 20.0 & 1.0 & 0.19 & 0.12 & 0.01 & 0.01 & 54.93 & 0.01 & 0.05 & 0.02 & 0.01 & 0.57 & 43.0 & 98.04 \\
\hline
\end{tabular}


Table 4 Average weighted mean of Chemical analysis of calcium carbonate samples from XRF results.

\begin{tabular}{|c|c|c|c|c|c|c|c|c|c|c|c|c|c|c|c|c|}
\hline \multicolumn{2}{|c|}{ Location } & \multirow{2}{*}{ 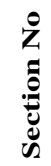 } & \multirow{2}{*}{ 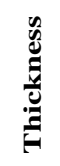 } & \multirow{2}{*}{$\begin{array}{l}0 \\
0 \\
0 \\
\dot{n}\end{array}$} & \multirow{2}{*}{$\begin{array}{l}0 \\
0 \\
0 \\
z\end{array}$} & \multirow{2}{*}{ 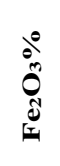 } & \multirow{2}{*}{$\begin{array}{l}0 \\
0 \\
\xi \\
\Sigma\end{array}$} & \multirow{2}{*}{$\begin{array}{l}\infty \\
0 \\
\sum^{\infty}\end{array}$} & \multirow{2}{*}{$\begin{array}{l}0 \\
0 \\
0 \\
0\end{array}$} & \multirow{2}{*}{$\begin{array}{l}80 \\
0 \\
\tilde{Z} \\
\tilde{Z}\end{array}$} & \multirow{2}{*}{ 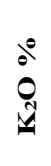 } & \multirow{2}{*}{$\begin{array}{l}b^{0} \\
0^{\prime \prime} \\
0\end{array}$} & \multirow{2}{*}{$\frac{8}{0}$} & \multirow{2}{*}{$\begin{array}{l}\delta \\
0 \\
\infty\end{array}$} & \multirow{2}{*}{$\begin{array}{l}\dot{0} \\
\dot{0} \\
\dot{0}\end{array}$} & \multirow{2}{*}{$\begin{array}{l}8 \\
0 \\
0 \\
0 \\
0\end{array}$} \\
\hline Lat. & Long. & & & & & & & & & & & & & & & \\
\hline $28^{\circ} 08^{\prime} 37^{\prime \prime}$ & $30^{\circ} 51^{\prime} 31^{\prime \prime}$ & 1 & 15.0 & 0.26 & 0.1 & 0.09 & 0.01 & 0.31 & 55.50 & 0.16 & 0.1 & $\begin{array}{c}0.00 \\
5\end{array}$ & 0.03 & 0.36 & 43.27 & 98.63 \\
\hline $28^{\circ} 02^{\prime} 58^{\prime \prime}$ & $30^{\circ} 50^{\prime} 34^{\prime \prime}$ & 2 & 12.0 & 0.15 & 0.1 & 0.09 & 0.01 & 0.30 & 55.22 & 0.13 & 0.1 & 0.01 & 0.1 & 0.1 & 43.61 & 98.55 \\
\hline $28^{\circ} 11^{\prime} 41^{\prime \prime}$ & $30^{\circ} 50^{\prime} 15^{\prime \prime}$ & 3 & 9.00 & 0.1 & 0.1 & 0.03 & 0.01 & 0.29 & 55.67 & 0.14 & 0.1 & 0.01 & 0.01 & 0.1 & 43.64 & 99.36 \\
\hline $28^{\circ} 10^{\prime} 21^{\prime \prime}$ & $30^{\circ} 47^{\prime} 34^{\prime \prime}$ & 4 & 33.0 & 1.0 & 0.06 & 0.03 & 0.01 & 0.01 & 55.52 & 0.01 & 0.02 & 0.02 & 0.01 & 0.01 & 43.43 & 99.09 \\
\hline $28^{\circ} 13^{\prime} 44^{\prime \prime}$ & $30^{\circ} 50^{\prime} 38^{\prime \prime}$ & 5 & 15.0 & 0.06 & 0.1 & 0.05 & 0.01 & 0.31 & 5541 & 0.24 & 0.1 & 0.01 & 0.06 & 0.14 & 43.44 & 98.90 \\
\hline $28^{\circ} 07^{\prime} 54^{\prime \prime}$ & $30^{\circ} 55^{\prime} 57^{\prime \prime}$ & 6 & 4.0 & 0.03 & 0.01 & 0.01 & 0.01 & 0.07 & 55.12 & 0.29 & 0.01 & 0.01 & 0.33 & 0.25 & 43.59 & 98.39 \\
\hline $28^{\circ} 04^{\prime} 11^{\prime \prime}$ & $30^{\circ} 53^{\prime} 19^{\prime \prime}$ & 7 & 3.0 & 0.02 & 0.01 & 0.01 & 0.01 & 0.07 & 55.26 & 0.15 & 0.01 & 0.01 & 0.17 & 0.63 & 43.42 & 98.64 \\
\hline $28^{\circ} 11^{\prime} 34^{\prime \prime}$ & $30^{\circ} 50^{\prime} 15^{\prime \prime}$ & 8 & 4.0 & 0.03 & 0.01 & 0.01 & 0.01 & 0.07 & 53.97 & 1.71 & 0.01 & 0.01 & 1.94 & 0.14 & 41.90 & 96.34 \\
\hline $28^{\circ} 11^{\prime} 16^{\prime \prime}$ & $30^{\circ} 49^{\prime} 08^{\prime \prime}$ & 9 & 4.0 & 0.02 & 0.01 & 0.01 & 0.01 & 0.06 & 54.80 & 0.88 & 0.01 & 0.01 & 0.99 & 0.12 & 42.80 & 97.82 \\
\hline $28^{\circ} 10^{\prime} 57^{\prime \prime}$ & $30^{\circ} 51^{\prime} 05^{\prime \prime}$ & 10 & 3.0 & 0.03 & 0.01 & 0.01 & 0.01 & 0.06 & 55.53 & 0.18 & 0.01 & 0.01 & 0.20 & 0.18 & 43.67 & 99.12 \\
\hline $28^{\circ} 14^{\prime} 03^{\prime \prime}$ & $30^{\circ} 51^{\prime} 59^{\prime \prime}$ & 11 & 4.0 & 0.02 & 0.01 & 0.01 & 0.01 & 0.07 & 55.78 & 0.12 & 0.01 & 0.01 & 0.14 & 0.03 & 43.58 & 99.57 \\
\hline $27^{0} 53^{\prime} 33^{\prime \prime}$ & $30^{\circ} 57^{\prime} 52^{\prime \prime}$ & 12 & 10.0 & 0.1 & 0.1 & 0.04 & 0.01 & 0.31 & 55.52 & 0.14 & 0.1 & 0.01 & 0.1 & 0.1 & 43.73 & 99.09 \\
\hline $28^{\circ} 06^{\prime} 06^{\prime \prime}$ & $30^{\circ} 59^{\prime} 33^{\prime \prime}$ & 13 & 8.0 & 0.1 & 0.1 & 0.03 & 0.01 & 0.41 & 55.52 & 0.12 & 0.1 & 0.01 & 0.1 & 0.04 & 43.66 & 99.09 \\
\hline $28^{\circ} 20^{\prime} 50^{\prime \prime}$ & $30^{\circ} 53^{\prime} 27^{\prime \prime}$ & 14 & 41.0 & 1.0 & 0.14 & 0.04 & 0.01 & 0.01 & 55.10 & 0.01 & 0.06 & 0.05 & 0.01 & 0.29 & 43.26 & 98.36 \\
\hline $28^{\circ} 07^{\prime} 10^{\prime \prime}$ & $30^{\circ} 47^{\prime} 04^{\prime \prime}$ & 15 & 71.0 & 1.00 & 0.28 & 0.14 & 0.01 & 0.01 & 55.41 & 0.01 & 0.01 & 0.30 & 0.01 & 0.01 & 43.25 & 98.90 \\
\hline $28^{\circ} 09^{\prime} 58^{\prime \prime}$ & $30^{\circ} 48^{\prime} 20^{\prime \prime}$ & 16 & 30.0 & 1.0 & 0.07 & 0.03 & 0.01 & 0.01 & 55.36 & 0.01 & 0.03 & 0.01 & 0.75 & 0.01 & 43.50 & 98.81 \\
\hline $27^{\circ} 57^{\prime} 24^{\prime \prime}$ & $31^{\circ} 10^{\prime} 45^{\prime \prime}$ & 17 & 34.0 & 1.0 & 0.13 & 0.04 & 0.01 & 0.01 & 55.47 & 0.01 & 0.04 & 0.03 & 0.01 & 0.01 & 43.55 & 99.01 \\
\hline $27^{\circ} 57^{\prime} 25^{\prime \prime}$ & $31^{\circ} 02^{\prime} 29^{\prime \prime}$ & 18 & 7.0 & 1.0 & 0.23 & 0.09 & 0.01 & 0.02 & 55.23 & 0.01 & 0.04 & 0.02 & 0.06 & 0.02 & 43.27 & 98.57 \\
\hline $28^{\circ} 14^{\prime} 52^{\prime \prime}$ & $30^{\circ} 47^{\prime} 20^{\prime \prime}$ & 19 & 6.0 & 0.01 & 0.1 & 0.05 & 0.01 & 0.32 & 55.66 & 0.13 & 0.1 & 0.01 & 0.1 & 0.1 & 43.55 & 99.34 \\
\hline $28^{\circ} 16^{\prime} 13^{\prime \prime}$ & $30^{\circ} 47^{\prime} 22^{\prime \prime}$ & 20 & 5.0 & 0,07 & 0.03 & 0.05 & 0.01 & 0.05 & 54.71 & 0.02 & 0.01 & 0.01 & 0.01 & 0.03 & 43.4 & 97.65 \\
\hline $28^{\circ} 18^{\prime} 14^{\prime \prime}$ & $30^{\circ} 47^{\prime} 21^{\prime \prime}$ & 21 & 12.0 & 0.1 & 0.1 & 0.04 & 0.01 & 0.33 & 54.97 & 0.15 & 0.1 & 0.01 & 0.07 & 0.36 & 42.37 & 98.18 \\
\hline $28^{\circ} 18^{\prime} 01^{\prime \prime}$ & $30^{\circ} 48^{\prime} 11^{\prime \prime}$ & 22 & 4.0 & 0.01 & 0.01 & 0.01 & 0.01 & 0.06 & 55.69 & 0.12 & 0.01 & 0.01 & 0.14 & 0.07 & 43.71 & 99.41 \\
\hline $28^{\circ} 17^{\prime} 51^{\prime \prime}$ & $30^{\circ} 47^{\prime} 35^{\prime \prime}$ & 23 & 14.0 & 0.1 & 0.1 & 0.03 & 0.01 & 0.32 & 55.68 & 0.14 & 0.1 & 0.01 & 0.1 & 0.14 & 43.63 & 99.11 \\
\hline $28^{\circ} 22^{\prime} 31^{\prime \prime}$ & $31^{\circ} 02^{\prime} 44^{\prime \prime}$ & 24 & 10.0 & 0.08 & 0.1 & 0.05 & 0.01 & 0.40 & 55.22 & 0.12 & 0.1 & 0.02 & 0.1 & 0.1 & 43.77 & 98.56 \\
\hline $28^{\circ} 20^{\prime} 51^{\prime \prime}$ & $30^{\circ} 47^{\prime} 54^{\prime \prime}$ & 25 & 17.0 & 1.0 & 0.1 & 0.03 & 0.01 & 0.01 & 55.45 & 0.01 & 0.02 & 0.02 & 0.01 & 0.01 & 43.50 & 98.96 \\
\hline $28^{\circ} 22^{\prime} 14^{\prime \prime}$ & $30^{\circ} 48^{\prime} 42^{\prime \prime}$ & 26 & 5.0 & 0.14 & 0.07 & 0.02 & 0.01 & 0.08 & 55.90 & 0.07 & 0.01 & 0.01 & 0.01 & 0.02 & 45.00 & 99.77 \\
\hline $28^{\circ} 22^{\prime} 03^{\prime \prime}$ & $31^{\circ} 07^{\prime} 03^{\prime \prime}$ & 27 & 35.0 & 0.16 & 0.1 & 0.05 & 0.01 & 0.29 & 56.09 & 0.13 & 0.1 & 0.04 & 0.1 & 0.1 & 43.15 & 99.99 \\
\hline $28^{\circ} 05^{\prime} 53^{\prime \prime}$ & $30^{\circ} 59^{\prime} 59^{\prime \prime}$ & 28 & 2.0 & 0.1 & 0.1 & 0.04 & 0.01 & 0.30 & 55.22 & 0.12 & 0.1 & 0.01 & 0.1 & 0.61 & 43.42 & 98.56 \\
\hline $28^{\circ} 17^{\prime} 09^{\prime \prime}$ & $30^{\circ} 59^{\prime} 10^{\prime \prime}$ & 29 & 20.0 & 1.0 & 0.19 & 0.12 & 0.01 & 0.01 & 54.93 & 0.01 & 0.05 & 0.02 & 0.01 & 0.57 & 43.0 & 98.04 \\
\hline
\end{tabular}

Figure $2(5-8)$ shows that, the average assays for $\mathrm{P}_{2} \mathrm{O}_{5} \%, \mathrm{Cl} \%, \mathrm{SO}_{3} \%$ were $0.02,0.15$ and 0.06 respectively, while, the loss in ignition (L.O.I) was 43.5\%.

Figure 2 (9-12) indicates that, the average assays of the following $\mathrm{Fe}_{2} \mathrm{O}_{3}, \mathrm{Al}_{2} \mathrm{O}_{3}, \mathrm{MgO}$ and $\mathrm{SiO}_{2} \%$ were $0.04,0.09,0.2$ and $0.3 \%$ respectively.

\section{Classification of the limestone by purity}

In comparison to the listed results of the analysis of limestones purity as shown in Table 2 above, the purity of El-Minya Governorate limestone deposits could be classified as very high purity, high purity, medium purity, low and impure as shown by the listed results in Table 5 [17].

As seen from the results indicated in Table 5, limestone deposits purity in the area under investigation varied from very high-to-high pure limestone.

\section{Brightness percentage measurements.}

Elected representative samples were prepared to measure brightness percentage of limestone deposits in study area and the results indicated as seen in Table 6.
As illustrated in Table 6 which gives a wide area of limestone deposit at El-Minya Governorate (EIMinya, Jabal AT Tayr Al Qibli, E and N- E AS Saririyyah) has brightness percent greater than $94 \%$, especially, at El-Minya area, whenever the brightness of limestone reached $95 \%$ with an average bed thickness of $16.2 \mathrm{~m}$

\section{Physical Characteristics}

The physical examination of limestone samples collected from the area under investigation indicated that, the limestone are free from chart, organic matter and pyrite, iron sulfides, quartz and uniform in texture and color. These physical characteristic allow limestone at El-Minya Governorate to be proper to many of commercial industrial uses.

\section{Limestone Industrial uses}

According to the obtained results, the limestone deposits are suitable for many of commercial industrial uses. Table 7 below shows deferent commercial industrial uses for limestone deposits located at El-Minya Governorate. 

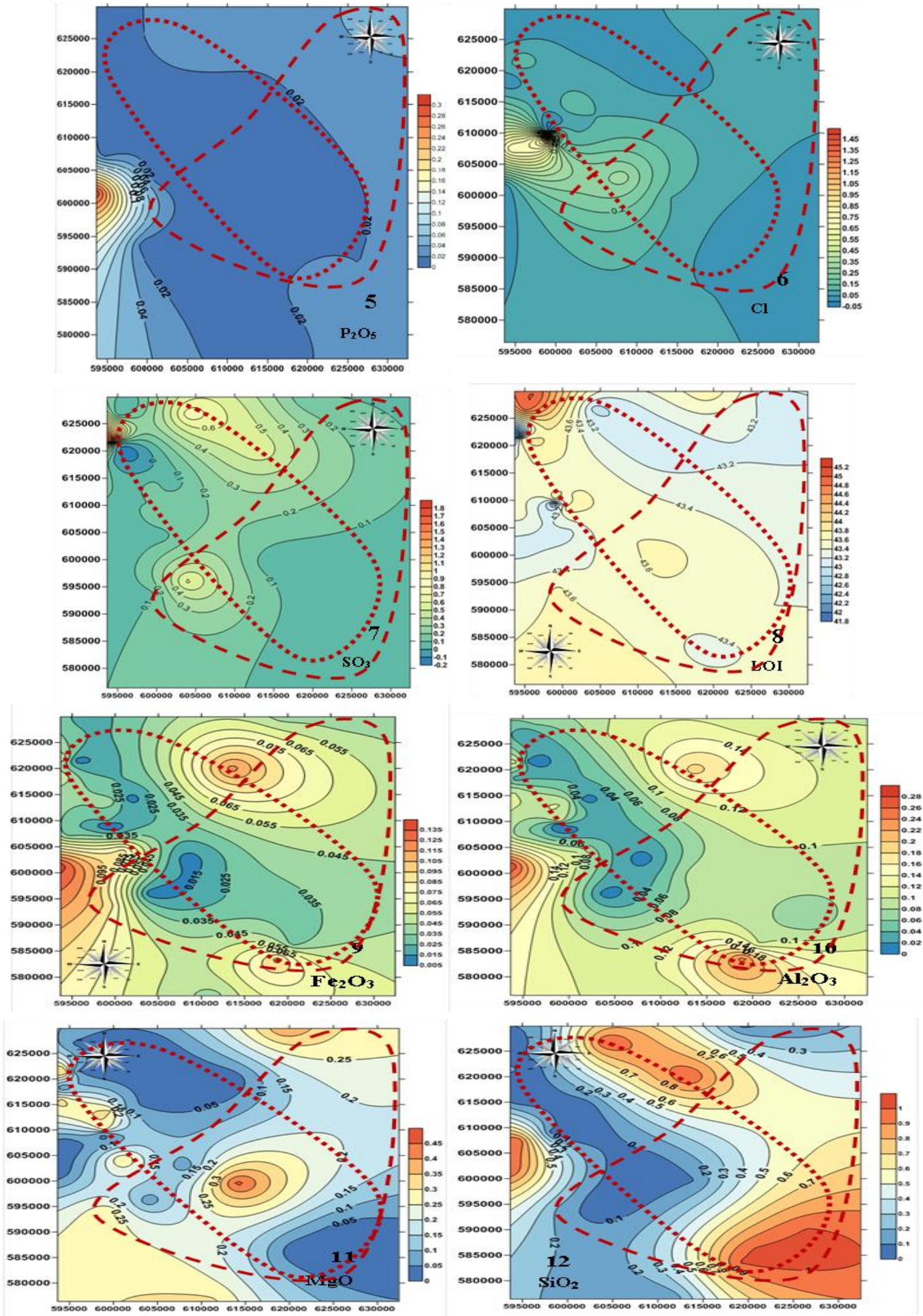

Figure 2 Assay contour map (1- $\mathrm{CaO} ; 2-\mathrm{CaCO}_{3} ; 3-\mathrm{K}_{2} \mathrm{O} ; 4-\mathrm{Na}_{2} \mathrm{O}$;5- $\mathrm{P}_{2} \mathrm{O}_{5} ; 6-\mathrm{Cl}$; $7-\mathrm{SO}_{3} ; 8-\mathrm{LOI} ; 9-\mathrm{Fe}_{2} \mathrm{O}_{3} ; 10-\mathrm{Al}_{2} \mathrm{O}_{3} ; 11-\mathrm{MgO}$; $\left.12-\mathrm{SiO}_{2}\right)$ 
Table 5 Limestone purity classification of the area of the study (El-Minya Governorate).

\begin{tabular}{|c|c|c|c|c|c|c|c|c|c|}
\hline & & & & & & & Category & & \\
\hline Lat. & Long. & $\stackrel{\nu}{ }$ & 产 & 0 & purity & purity & purity & purity & impure \\
\hline $28008^{\prime \prime} 37^{\prime \prime}$ & $30 \circ 51^{\prime} 31^{\prime \prime}$ & 1 & $\underline{35.0}$ & 99.99 & $x$ & -- & -- & -- & -- \\
\hline $28002^{\prime} 58^{\prime \prime}$ & $30=50^{\prime} 34^{\prime \prime}$ & 2 & 10.0 & 99.09 & $x$ & -- & -- & -- & -- \\
\hline 280 11'41" & $30=50^{\prime} 15^{\prime \prime}$ & 3 & 12.0 & 98.54 & -- & $x$ & -- & -- & -- \\
\hline $28010^{\prime} 21^{\prime \prime}$ & $30=47^{\prime} 34^{\prime \prime}$ & 4 & 8.00 & 99.15 & $x$ & -- & -- & -- & -- \\
\hline 28 ' $13^{\prime} 44^{\prime \prime}$ & $30=50^{\prime} 38^{\prime \prime}$ & 5 & 15.0 & 98.88 & -- & $x$ & -- & -- & -- \\
\hline $28=07^{\prime} 54^{\prime \prime}$ & $30 \circ 55^{\prime} 57^{\prime \prime}$ & 6 & 9.00 & 99.36 & $\mathrm{X}$ & -- & -- & -- & -- \\
\hline $28004^{\prime} 11^{\prime \prime}$ & $30 \circ 53^{\prime} 19^{\prime \prime}$ & 7 & 15.0 & 98.68 & $x$ & -- & -- & -- & -- \\
\hline 280 11'34" & $30=50^{\prime} 15^{\prime \prime}$ & 8 & 6.00 & 99.34 & $x$ & -- & -- & -- & -- \\
\hline 280 11' $16^{\prime \prime}$ & $30 \circ 49^{\prime} 08^{\prime \prime}$ & 9 & $\underline{14.0}$ & 99.38 & $x$ & -- & -- & -- & -- \\
\hline $28=10^{\prime} 57^{\prime \prime}$ & $30=51^{\prime} 05^{\prime \prime}$ & 10 & 12.0 & 98.16 & -- & $x$ & -- & -- & -- \\
\hline 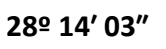 & $30=51^{\prime} 59^{\prime \prime}$ & 11 & 10.0 & 98.56 & $x$ & -- & --- & -- & -- \\
\hline $27^{\circ} 53^{\prime} 33^{\prime \prime}$ & $30=57^{\prime} 52^{\prime \prime}$ & 12 & 20.0 & 98.04 & -- & $x$ & -- & -- & -- \\
\hline $28 \div 06^{\prime} 06^{\prime \prime}$ & $30=59^{\prime} 33^{\prime \prime}$ & 13 & 41.0 & 98.34 & -- & $x$ & -- & -- & -- \\
\hline $28 \circ 20^{\prime} 50^{\prime \prime}$ & $30 \circ 53^{\prime} 27^{\prime \prime}$ & 14 & $\underline{71.0}$ & 98.54 & -- & $x$ & -- & -- & -- \\
\hline $2807^{\prime} 10^{\prime \prime}$ & $30 \circ 47^{\prime} 04^{\prime \prime}$ & 15 & 33.0 & 99.11 & -- & $x$ & -- & -- & -- \\
\hline $28009^{\prime} 58^{\prime \prime}$ & $30 \circ 48^{\prime} 20^{\prime \prime}$ & 16 & 30.0 & 98.81 & -- & $x$ & -- & -- & -- \\
\hline $27^{\circ} 57^{\prime \prime} 24^{\prime \prime}$ & $31010^{\prime} 45^{\prime \prime}$ & 17 & 34.0 & 99.00 & -- & $x$ & -- & -- & -- \\
\hline "27임 $57^{\prime \prime}$ & $31002^{\prime} 29^{\prime \prime}$ & 18 & 7.0 & 98.54 & -- & $x$ & -- & -- & -- \\
\hline 28 - 14' 52" & $30 \circ 47^{\prime} 20^{\prime \prime}$ & 19 & 17.0 & 98.95 & -- & $x$ & -- & -- & -- \\
\hline $28016^{\prime} 13^{\prime \prime}$ & $30=47^{\prime} 22^{\prime \prime}$ & 20 & 4.0 & 97.65 & -- & $x$ & -- & -- & -- \\
\hline 28 18 $18^{\prime \prime}$ & $30 \circ 47^{\prime} 21^{\prime \prime}$ & 21 & $\underline{5.0}$ & 99.77 & $\mathrm{X}$ & -- & -- & -- & -- \\
\hline $28=18^{\prime} 01^{\prime \prime}$ & $30048^{\prime} 11^{\prime \prime}$ & 22 & 4.0 & 99.57 & $x$ & -- & -- & -- & -- \\
\hline 28 17' 51" & $30=47^{\prime} 35^{\prime \prime}$ & 23 & 5.0 & 97.65 & -- & $x$ & -- & -- & -- \\
\hline $28022^{\prime} 31^{\prime \prime}$ & $31 \circ 02^{\prime} 44^{\prime \prime}$ & 24 & 4.0 & 99.41 & $x$ & -- & -- & -- & -- \\
\hline $28020^{\prime} 51^{\prime \prime}$ & $30047^{\prime} 54^{\prime \prime}$ & 25 & 4.0 & 98.39 & -- & $x$ & -- & -- & -- \\
\hline $28=22^{\prime} 14^{\prime \prime}$ & $30=48^{\prime} 42^{\prime \prime}$ & 26 & 3.0 & 98.64 & $X$ & -- & -- & -- & -- \\
\hline 28 o $22^{\prime} 03^{\prime \prime}$ & $31 \circ 07^{\prime} 03^{\prime \prime}$ & 27 & 4.0 & 96.34 & -- & -- & $x$ & -- & -- \\
\hline $28005^{\prime} 53^{\prime \prime}$ & $30 \circ 59^{\prime} 59^{\prime \prime}$ & 28 & 4.0 & 97.82 & -- & $x$ & -- & -- & -- \\
\hline 28 ' $17^{\prime \prime} 09^{\prime \prime}$ & $30=59^{\prime} 10^{\prime \prime}$ & 29 & 3.0 & 99.12 & $x$ & -- & --- & -- & -- \\
\hline
\end{tabular}

Table 6 Brightness percent in the study area at El-Minya Governorate.

\begin{tabular}{|c|c|c|c|c|c|c|c|c|c|}
\hline \multirow{2}{*}{\multicolumn{3}{|c|}{ Location }} & \multirow{3}{*}{ 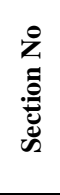 } & \multirow{3}{*}{ 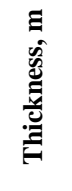 } & \multirow{2}{*}{ Oீ } & \multicolumn{2}{|c|}{ Category } & \multicolumn{2}{|c|}{ Brightness \% } \\
\hline & & & & & & & & & \\
\hline Lat. & Long. & Area & & & & $\begin{array}{c}\text { Very high } \\
\text { purity }\end{array}$ & $\begin{array}{l}\text { High } \\
\text { purity }\end{array}$ & $\begin{array}{c}\text { Brightness } \\
\%\end{array}$ & $\begin{array}{c}\text { Average } \\
\%\end{array}$ \\
\hline $28^{\circ} 08^{\prime} 37^{\prime \prime}$ & $30^{\circ} 51^{\prime} 31^{\prime \prime}$ & \multirow{5}{*}{ El-Minya } & G1 & 15.0 & 98.88 & -- & $\mathbf{X}$ & 94.67 & \multirow{5}{*}{95.00} \\
\hline $28^{\circ} 02^{\prime} 58^{\prime \prime}$ & $30^{\circ} 50^{\prime} 34^{\prime \prime}$ & & G2 & 12.0 & 98.54 & -- & $\mathbf{X}$ & 94.28 & \\
\hline $28^{\circ} 11^{\prime} 41^{\prime \prime}$ & $30^{\circ} 50^{\prime} 15^{\prime \prime}$ & & G3 & 9.00 & 99.36 & $\mathbf{X}$ & -- & 94.47 & \\
\hline $28^{\circ} 10^{\prime} 21^{\prime \prime}$ & $30^{\circ} 47^{\prime} 34^{\prime \prime}$ & & G4 & 30.0 & 98.81 & -- & $\mathbf{X}$ & 95.36 & \\
\hline $28^{\circ} 13^{\prime} 44^{\prime \prime}$ & $30^{\circ} 50^{\prime} 38^{\prime \prime}$ & & G5 & 15.0 & 98.68 & $\mathbf{X}$ & -- & 95.39 & \\
\hline $28^{\circ} 14^{\prime} 52^{\prime \prime}$ & $30^{\circ} 47^{\prime} 20^{\prime \prime}$ & \multirow{3}{*}{$\begin{array}{c}\text { Jabal AT Tayr } \\
\text { Al Qibli }\end{array}$} & G6 & 6.00 & 99.34 & $\mathbf{X}$ & -- & 95.48 & \multirow{3}{*}{94.25} \\
\hline $28^{\circ} 16^{\prime} 13^{\prime \prime}$ & $30^{\circ} 47^{\prime} 22^{\prime \prime}$ & & G7 & 5.0 & 97.65 & -- & $\mathbf{X}$ & 94.93 & \\
\hline $28^{\circ} 18^{\prime} 14^{\prime \prime}$ & $30^{\circ} 47^{\prime} 21^{\prime \prime}$ & & G8 & 12.0 & 98.16 & -- & $\mathbf{X}$ & 93.35 & \\
\hline $28^{\circ} 20^{\prime} 51^{\prime \prime}$ & $30^{\circ} 47^{\prime} 54^{\prime \prime}$ & \multirow{2}{*}{$\begin{array}{c}\text { E and N-E AS } \\
\text { Saririyyah }\end{array}$} & G9 & 4.0 & 97.65 & -- & $\mathbf{X}$ & 92.87 & \multirow{2}{*}{94.33} \\
\hline $28^{\circ} 22^{\prime} 14^{\prime \prime}$ & $30^{\circ} 48^{\prime} 42^{\prime \prime}$ & & G10 & 5.0 & 99.77 & $\mathbf{x}$ & -- & 95.50 & \\
\hline \multicolumn{9}{|c|}{ Average } & 94.79 \\
\hline
\end{tabular}


Table 7 Commercial industrial uses for limestone deposits located at El-Minya Governorate.

\begin{tabular}{|c|c|c|c|c|c|c|c|c|c|c|c|c|c|}
\hline \multirow{3}{*}{ Area } & \multirow{3}{*}{ 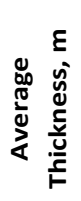 } & \multirow{3}{*}{ 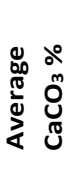 } & \multirow{3}{*}{$\begin{array}{c}\text { Average } \\
\text { Brightness \% }\end{array}$} & \multirow{3}{*}{ Category } & \multicolumn{9}{|c|}{ Uses } \\
\hline & & & & & \multirow{2}{*}{$\begin{array}{l}\text { Building } \\
\text { stone }\end{array}$} & \multicolumn{8}{|c|}{ Industrial uses* } \\
\hline & & & & & & 1 & 2 & 3 & 4 & 5 & 6 & 7 & 8 \\
\hline El-Minya & 16 & 98.82 & 95.00 & $\begin{array}{l}\text { High to } \\
\text { Very high } \\
\text { purity }\end{array}$ & v & v & v & v & v & v & v & v & v \\
\hline $\begin{array}{c}\text { Jabal AT Tayr Al } \\
\text { Qibli }\end{array}$ & 7.7 & 98.36 & 94.25 & High purity & v & v & v & v & v & v & v & v & v \\
\hline $\begin{array}{c}\text { E and N-E AS- } \\
\text { Saririyyah }\end{array}$ & 4.5 & 98.83 & 94.33 & $\begin{array}{l}\text { High to } \\
\text { Very high } \\
\text { purity }\end{array}$ & $\checkmark$ & $\sqrt{ }$ & v & v & v & $\begin{array}{lll}v & -a\end{array}$ & 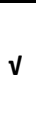 & v & $\sqrt{ }$ \\
\hline
\end{tabular}

"1- Paint; 2- Paper; 3- Plastic; 4- Food \& pharmaceutic; 5- Ceramic; 6- Rubber; 7-Adhesives \& sealants; 8- Agriculture \& animal feed

\section{Conclusions}

From the achieved results, it could be concluded that:

- The limestone deposits located at El-Minya Governorate Areas (El-Minya, Jabal AT Tayr Al Qibli, E and N- E AS-Saririyyah) could be considered as potential source of high-grade calcium carbonate.

- The evaluated limestone deposits have chemical and physical properties are proper for different industrial uses such as Paint, Paper, Plastic, Food \& Pharmaceutic, Ceramic, Rubber, Adhesives \& Sealants an Agriculture \& Feed.

- Orientation of limestone deposits to the industrial uses instead of building materials increase its benefit and value.

\section{REFERENCES}

[1] D.S. Rao, T.V. Vijayakumar, S. Prabhakar, and G. Bhaskar Raju, Geochemical assessment of a siliceous limestone sample for cement making, Chin. J. Geochem. 30; 2011, pp 033-039.

[2] S. Chatterjee, A. Bhattacherjee, B. Samanta, and S.K. Pal, Ore Grade Estimation of a Limestone Deposit in India Using an Artificial Neural Network, Applied GIS, Volume 2, Number 1, Monash University E press, 2006, pp 3.1-3.20.

[3] Web site: www.ima-eu.org/cca.html.

[4] C. Mitchell, Reconnaissance assessment of high-purity limestone, Industrial Minerals Specialist, British Geological Survey, Nottingham, NG12 5GG, UK.

[5] High purity limestone questm industrial minerals, December 2011, pp 48-52.

[6] British Geological Survey, Mineral Planning Factsheet: Limestone, 9; 2006.
[7] www.bgs.ac.uk/mineralsuk/downloads/mpflimestone pdf.

[8] Scientific Opinion on re-evaluation of calcium carbonate (E 170) as a food Additive, EFSA Panel on Food Additives and Nutrient Sources added to Food (ANS), EFSA Journal, 9, 7; 2011, 2318.

[9] Temidayo Bolarinwa1, S. Ojochogwu Idakwo and A. Tope Shade, Economic Aspects of Carbonates of the Albian Asu River Group in Tse-Kucha Near Yandev, Middle Benue Trough, Nigeria, Journal of Geography and Geology; Vol. 5, No. 2; 2013, pp 85-98.

[10] F. H. A. El-Rahiem, M. A. Arafa and M. M. Farhat, "Flotation of fine particles of Egyptian ground calcium carbonate as added value of filler in some industries", Mineral Processing and Extractive Metallurgy (Trans. Inst. Min Metall. C) ,Vol 115 , No 3; 2006, pp. 161 164.

[11] A.M. Ali Mahrous and Hyung-Sik Yang, A study of some Egyptian carbonate rocks for the building construction industry, International Journal of Mining Science and Technology, 24; 2014, 467-470.

[12] Sayed Mohammad, A, El-Sageer Hassan and Y. Hussein Mamdouh, Evaluation of the engineering properties of some Egyptian limestone as controlling factors for highways construction and cement industry. In: The $3^{\text {rd }}$ International Conference for Building and Construction. Cairo; 1996. p. 323-32.

[13] MI. Sayed Ahmed and AA. Abdel Wahab, Geotechnical properties of some middle Eocene limestone along the Nile valley. Egypt J Geol, 27(1-2), 1983, pp 27-42.

[14] M. Ali Mahrous, M. El-Beblawi Mostafa, A. Y. Mohamed, H. El-Sageer and T. Mostafa, Design and planning of some building materials quarries for different purposes in Sohag and Quena Governorates, Egypt: Assiut University, 2007.

[15] M. Mahrous, M. Mostafa Tantawi and H. El-Sageer. Evaluation of the engineering properties of some Egyptian limestones as construction materials for 
highway pavements. Constr Build Mater, 24; 2010, pp 2598-2603.

[16] J. lannicelli, and H. H. Murray, "A Survey-Beneficiation of Industrial Minerals, Ores, and Coal by High Intensity Wet Magnetic Separation”, NSF / RA- 800286, 1989.

[17] D.J. Harrison, Industrial minerals laboratory manual (limestone), British Geo. Sarv. Technical Report WG/92/29, 1992.

[18] M. S. Abu El Ghar and A.W. Hussein, Post-Depositional Changes of the Lower-Middle Eocene Limestones of the Area between Assiut and Minia, West of the Nile Valley, Egypt, First International Conference on the Geology of the Tethys, Cairo University, November, 2005.

[19] S. Omara, Mansour, H., M. Youssef and H. Khalifa, Stratigraphy, paleoenvironment and structural features of the area east of Bani Mazar-Upper Egypt; Bull. Fac. Science, Assuit Univ., Egypt, Vol. 6, No. (S), 1977, pp. 171-197.

[20] S. Omara, E.R. Philibbos and S., Hanna, Contribution to the geometry and geology of the area east El Minia; Bull. Boc., Geography, Egypt, 1976.

[21] R. Said, Explanatory note to accompany of Geological map of Egypt, Geol. Surv.,Cairo, Egypt, paper 56, 1971, pp. 123.

[22] A. Sheleiby and M.A. Eid, Stratigraphy of the Paleogene sediments at El Galala El Qebliya, Ginient El Atash and Wadi Tarfa between long. $31^{\circ} 00^{\prime}-32^{\circ} 30^{\prime}$ $\mathrm{E}$ and $28^{\circ} 00^{\prime}-29^{\circ} 00^{\prime} \mathrm{N}$, internal report no. 10/1996, EGSMA, Cairo, Egypt, 1996.

[23] A. Sheleiby and M.A. Eid, Geology and lithostratigraphy of the area east and NE- Assuit, internal report no. 7/2000, EGSMA, Cairo, Egypt, 2000. 